BARBARA LÜSCHER

\title{
Zwei interessante Fälschungen
}

\author{
Hierzu Tafel II-III
}

Von einer Ägyptenreise im Herbst 1983 brachte ich einen sehr qualitätvoll gefälschten Skarabäus mit, der auf seiner Basisfläche acht Schriftzeilen trägt sowie verschiedene Darstellungen auf der Rückenpartie. Knapp einen Monat später brachte mir ein Kollege einen zweiten fast identischen Skarabäus aus Ägypten mit, vom gleichen Händler.

Der Grund, warum ich diesen eindeutig gefälschten Stücken einen Artikel widme, liegt darin, daß sich die vom Fälscher für die Schriftzeilen benutzte Vorlage bestimmen läßt. Dies ist umso interessanter, als bereits seit längerer Zeit ein nach derselben Vorlage gefälschter Skarabäus bekannt ist. Doch zuvor eine kurze äußere Beschreibung der beiden Skarabäen:

Skarabäus A (Taf. IIa, IIIa)

Skarabäus B (Taf. IIb, IIIb)

Länge: $3,96 \mathrm{~cm}$

Länge : $3,44 \mathrm{~cm}$

Breite: $3,03 \mathrm{~cm}$

Breite: $2,48 \mathrm{~cm}$

Höhe: 1,21 cm

Höhe: $1,68 \mathrm{~cm}$

Beide sind aus demselben dunkelbraunen Material gearbeitet, unglasiert, ohne Durchbohrung. Die Schriftzeilen auf der Basisfläche sind in sehr kleinen Hieroglyphen eingetieft.

Sie stammen beide vom selben Händler beim Tempel Sethos' I. in Abydos.

Bei der Inschrift nun, die jeweils in acht waagerechten Zeilen auf der Basisfläche der Skarabäen angebracht ist, handelt es sich um den Text über die Löwenjagd, die Amenophis III. auf über 140 sogenannten Gedenkskarabäen verewigen ließ (vgl. dazu die grundlegende Arbeit von C. Blanken berg - Van Delden, The Large Commemorative Scarabs of Amenhotep III. Leiden 1969). Von den fünf bekannten Serien (,Teje“, „Wildstierjagd“, „Löwenjagd“, „Gilullepa“, ,Teichanlage“), alle aus den ersten elf Regierungsjahren des Königs, ist der Text der Löwenjagd weitaus am häufigsten vertreten. Dabei gleicht kaum ein Exemplar genau dem anderen, da die Stücke einzeln und nicht in Serie angefertigt wurden. Auch ist der Text recht unterschiedlich auf die meist acht zur Verfügung stehenden Zeilen verteilt, ebenso gibt es zum Teil Abweichungen in der Schreibung einzelner Worte.

Bei den beiden Fälschungen jedoch ist die Anordnung, Verteilung und Zeichenform der Hieroglyphen bis in kleinste Ungenauigkeiten hinein nahezu identisch; allerdings mit Ausnahme der Kartuschen, wovon noch zu sprechen sein wird.

Diese exakte Übereinstimmung veranlaßte mich, unter den bisher bekannten bzw. publizierten echten Exemplaren nach einer Entsprechung zu suchen. Dabei stieß ich bei einem Nachtrags-Artikel von Frau Blankenberg-Van Delden (JEA 62, 1976, S. 75) auf folgende Bemerkung:

„No fakes would have been incorporated into this article were it not that for the first time the scarab or rather the reproduction of the scarab that served as a model is known. This reproduction can be found in 'The Timins collection of Ancient Egyptian Scarabs and Cylinder Seals' (London 1907), pI. XXI, no. 16, by P. E. Newberry. The authentic scarab is in the Metropolitan Museum of Art, New York (no. C80).“

Und von genau derselben Reproduktion scheint nun auch unser Fälscher abgeschrieben zu haben. Bei einem Vergleich der Reproduktion aus dem Timins-Katalog (Fig. 1) mit den Fotos der beiden Fälschungen (Taf. IIa, b) zeigt sich nämlich nicht nur eine genaue Übereinstimmung der Zeichenanordnung und fast aller Zeichenformen, sondern der Fälscher hat offenbar sogar Striche, die nur eine Zerstörung oder Einkerbung wiedergeben, blind kopiert (man vergleiche dazu etwa die 


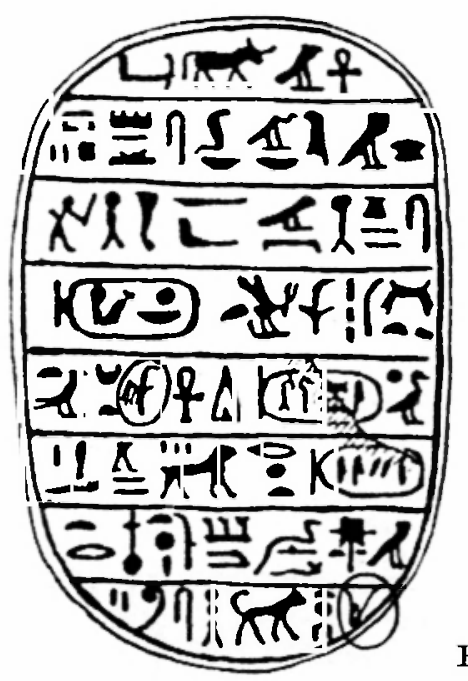

Fig. 1

beiden von mir durch einen Kreis auf der Reproduktion markierten Stellen 5 , r mit den Fotos). Das zeigt auch eindeutig, da $B$ der Fälscher von der Reproduktion abgeschrieben haben muß und nicht etwa von dem Foto des Originals.

Einzig bei den Kartuschen hat er sich nicht an die Vorlage gehalten. Auf Skarabäus A ersetzte erdie Namen von Amenophis III. und seiner Gemahlin Teje durch die Kartuschen (

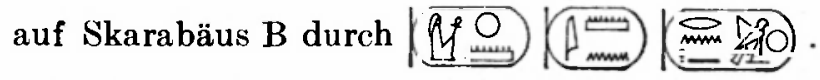

Doch auch hier läßt sich die Quelle zumindest erahnen. Diese Königsnamen, willkürlich an Stelle der echten Kartuschen gesetzt, sind doch recht erstaunlich. Da erscheinen die Thronnamen von Sethos I. und Ramses II., $M n-m 3^{e} t-R^{r}$ und $W s r-m 3^{r} t-R^{r}-s t p-n-R^{r}$, neben $M n j, N t r j-k 3-R^{r}$ und $M r j-n-H r w\left(-R^{c}\right.$ ?) (vgl. zu letzterem die deutlichere Schreibung auf der Rückenplatte von Skarabäus B, Taf. Ill b). Der Fälscher hatte offenbar auch diese Namen irgendwo abgeschrieben. Da beide Stücke in Abydos angeboten wurden, könnte man vermuten, da $B$ sich der Fälscher auch dort vom häufigen Auftreten der Kartuschen Sethos' I. und Ramses' II. inspirieren ließ. Vielleicht läßt es sich aber noch enger eingrenzen. Eine besondere Anhäufung von Königskartuschen bietet die bekannte Königsliste im Tempel Sethos' I. (vgl. z. B. die Strichzeichnung in ZÄS 2, 1864 Beilage hinten), und bezeichnenderweise finden sich dort neben dem oft wiederholten Namen Sethos' I. $M n-m 3^{e} t-R^{e}$ auch die Kartuschen mit den Namen $M n j$, Ntrj-kz- $R^{e}$ und $M r j-n-H r w$ (vom Fälscher offenbar noch durch eine Sonnenscheibe ergänzt). So liegt die Vermutung nahe, daß diese Königgliste als Vorlage für die in dem Text ausgetauschten Kartuschen diente.

Zu erwähnen bleibt noch, daß die Rückenpartien beider Skarabäen jeweils unterschiedlich dekoriert sind mit Götterdarstellungen, religiösen Emblemen und kurzen Beischriften (vgl. Taf. IIIa, b). Es handelt sich um Zusammenstellungen verschiedener entlehnter Motive, die aber nicht ein und derselben Quelle entnommen sein müssen.

Es ist anzunehmen, daß noch mehr Fälschungen gleicher Art in Umlauf sind. So teilte mir Dr. R. J. Demaree 1984 brieflich mit, daß ihm kürzlich von einem Sammler Fotos eines fast identischen Stückes zugeschickt wurden.

\section{NACHTRAG}

Nach Einsendung des Artikels wurde mir von einem Privatmann aus Bern ein Skarabäus vorgelegt, der in Machart und Dekoration der Basisfläche sowie der Rückenpartie praktisch identisch ist mit dem von mir als „Skarabäus A“ bezeichneten Stück. Er ist jedoch um wenige Millimeter größer, und die Gravur erscheint etwas frischer und schärfer. Erworben wurde er im Winter 1988 in Gurnalı. 